\title{
The use of a geographic information system to identify a dairy goat farm as the most likely source of an urban Q-fever outbreak
}

\author{
Barbara Schimmer ${ }^{1}$, Ronald ter Schegget ${ }^{2}$, Marjolijn Wegdam³ ${ }^{3}$ Lothar Züchner ${ }^{4}$, Arnout de Bruin ${ }^{1}$, \\ Peter M Schneeberger ${ }^{5}$, Thijs Veenstra ${ }^{1}$, Piet Vellema ${ }^{6}$, Wim van der Hoek ${ }^{1 *}$
}

\begin{abstract}
Background: A Q-fever outbreak occurred in an urban area in the south of the Netherlands in May 2008. The distribution and timing of cases suggested a common source. We studied the spatial relationship between the residence locations of human cases and nearby small ruminant farms, of which one dairy goat farm had experienced abortions due to Q-fever since mid April 2008. A generic geographic information system (GIS) was used to develop a method for source detection in the still evolving major epidemic of Q-fever in the Netherlands.

Methods: All notified Q-fever cases in the area were interviewed. Postal codes of cases and of small ruminant farms (size $>40$ animals) located within 5 kilometres of the cluster area were geo-referenced as point locations in a GIS-model. For each farm, attack rates and relative risks were calculated for 5 concentric zones adding 1 kilometre at a time, using the 5-10 kilometres zone as reference. These data were linked to the results of veterinary investigations.
\end{abstract}

Results: Persons living within 2 kilometres of an affected dairy goat farm ( $>400$ animals) had a much higher risk for Q-fever than those living more than 5 kilometres away (Relative risk 31.1 [95\% Cl 16.4-59.1]).

Conclusions: The study supported the hypothesis that a single dairy goat farm was the source of the human outbreak. GIS-based attack rate analysis is a promising tool for source detection in outbreaks of human Q-fever.

\section{Background}

Q-fever, a zoonosis caused by the bacterium Coxiella burnetii, is an emerging public health problem in the Netherlands. In May 2007, the first community Q-fever outbreak was documented around a single village in the province of Noord Brabant. A case control study carried out subsequently suggested a role of warm, dry weather conditions in addition to a residence location close to ruminant farms [1].

The unprecedented magnitude ( $>3000$ notified human cases) and geographical spread within the province during the following years (2008-2009) suggested multiple sources of infection [2]. The affected area matches with the part of the Netherlands that has the highest

\footnotetext{
* Correspondence: wim.van.der.hoek@rivm.nl

${ }^{1}$ Centre for Infectious Disease Control, National Institute for Public Health and the Environment, A van Leeuwenhoeklaan 9, PO Box 1, 3720 BA Bilthoven, the Netherlands
}

concentration of dairy goat farms, some of which are very large (>5000 goats) [3]. From 2005 to 2008, clinical Q-fever represented by abortions or stillbirths in small ruminants, was diagnosed at 22 large ( $>50$ goats) commercial dairy goat farms and 2 dairy sheep farms, mainly in the affected province [4]. Although large dairy goat farms were implicated, questions about exact transmission routes and risk factors remained unanswered.

In May 2008 the municipal health service (MHS) in the southeast region of the affected province was alerted by a cluster of human Q-fever cases in an urban area (88,000 inhabitants). Based on the distribution of cases in time and place, a common source was suspected. The MHS had been informed by the Animal Health Service that a dairy goat farm was diagnosed with Q-fever in their region. Some patients that were interviewed by the MHS indicated a pet farm in their neighbourhood as a potential source. 
The objective of the study was to assess whether the cluster of human Q-fever cases in this urban area could be linked to the suspected source on the basis of the distribution of illness onset and residence location relative to small ruminant farms. Furthermore, the study explored the usefulness of a generic geographic information system (GIS) for source detection in the still evolving major epidemic of Q-fever in the Netherlands.

\section{Methods}

\section{Human Q-fever cases}

Q-fever is a notifiable disease in the Netherlands and the regional MHS was responsible for the outbreak investigation. A regional laboratory provided the majority of microbiological diagnostic services for the inhabitants of the MHS region. Submitted serum samples were screened with a $C$. burnetii complement fixation test (CFT, Siemens, the Netherlands). Notification criteria for acute Q-fever were a clinical presentation with at least fever, or pneumonia, or hepatitis and a fourfold rise in titre in the CFT or a single high titre or a high titre in two samples without a fourfold increase (CFT titre $\geq 1: 4)$. All persons notified to the Dutch national surveillance system for infectious diseases (OSIRIS) who were residing or had visited the MHS region of Brabant Southeast and who had an illness onset from week 16 (14 April 2008) to week 32 (10 August 2008) were included.

A hypothesis generating questionnaire was used to get insight into possible sources of $C$. burnetii infection. Questions pertained to age, sex, day of illness onset, self-reported symptoms and complications, and a range of potential risk factors, such as travel history and occupational exposure. A recall period of four weeks before illness onset was used to investigate if cases had had relevant exposures immediately before the incubation period. Questionnaires to all notified cases were administered by trained public health employees from the MHS during house visits, or self-administered by mail.

Data on human cases was collected as part of the routine system of infectious disease surveillance and outbreak control. Because no additional information or laboratory materials were collected from patients, a medical ethical review was not needed.

\section{Meteorological data}

We determined the predominant wind direction by day and calculated the number of days that the wind had blown in every wind direction during the estimated exposure period. These data were available for weather station Volkel (station 375 of the Royal Netherlands Meteorological Institute), located within 15 kilometres from the centre of the cluster area.

\section{Veterinary data}

Veterinary data were obtained from the Animal Health Service. A mandatory veterinary notification system was only introduced on 12 June 2008 (week 24). If a veterinary source was suspected based on the exposure information from patients, the MHS notified the Food and Consumer Product Safety Authority.

\section{Data analysis}

Residence locations of all Q-fever cases were plotted as point data on a digital map of the area. The Animal Health Service provided the locations and specifications of goat and sheep farms that were located within 5 kilometres of the average longitudinal and lateral coordinate of the cases, thus defining the centre of the cluster. The residential addresses of the cases was considered a proxy for level of exposure to small ruminant farms. We hypothesized that the attack rate would be higher for persons living close to the source and would decrease with increasing distance. Around each farm with more than 40 small ruminants we made concentric rings each expanding with a 1 kilometre radius (range 0-5000 meters) and a reference ring with a range from 5000 10,000 meters). Attack rates for residents living within these zones were calculated using a digital map of population distribution. Relative risks for 5 zones were estimated using the 5000-10,000 meters zone as reference. Mapping and spatial analyses were done with ArcGis 9 software (ESRI, Redlands, CA, USA).

\section{Results}

Between week 16 and 33 (14 April-15 August 2008) the MHS Brabant Southeast notified 96 Q-fever cases. In 55 patients positive CFT titres were found in two sequential ( $\geq 14$ days interval) samples (of which 42 cases had a fourfold increase in titre) and 41 patients had a single positive CFT titre. The median age of cases was 53 years (interquartile range 42-61) and the male to female ratio was 2.3:1. Day of onset of illness was known for 95 cases (median 4 June 2008). Nineteen individuals (20\%) were hospitalised.

Eighty one (84\%) of the 96 cases completed a questionnaire. Most commonly reported symptoms were fever $>38^{\circ} \mathrm{C}(95 \%)$, fatigue $(83 \%)$, headache $(70 \%)$, night sweats (62\%), dyspnoea (53\%), malaise (52\%), and myalgia (51\%). The majority of cases (79\%) presented clinically with a pneumonia. Five patients (6\%) presented with hepatitis. Seventy eight $(81 \%)$ of the 96 cases that were notified in the MHS region Brabant Southeast resided in one city with 87,752 inhabitants and a surface area of $54.56 \mathrm{~km}^{2}$ $\left(1608 / \mathrm{km}^{2}\right)$. Eight cases were from adjacent municipalities and 10 from more distant municipalities and none of these reported to have a work address within the city. 
We estimated from the epidemic curve (Figure 1) that the likely period of infection was from week 15 (mid-April) to week 24 (mid-June). This encompassed the known two to four week incubation period for C. burnetii and was based on date of symptom onset for 95 of the 96 cases.

\section{Veterinary investigations}

There were 60 locations with small ruminants within the 5 kilometre zone around the centre of the human Qfever cluster. Seven of these locations were large farms (A-G) with more than 40 small ruminants, including one large dairy goat farm (A, >400 goats), one mixed sheep/ goat farm (E, 126 goats and 90 sheep) and 5 sheep farms (B, C, D, F, and G with between 57 and 118 sheep). These farms are mapped in figure 2. Farm A reported an abortion wave on 21 April 2008 (week 17) to the Animal Health Service and submitted two aborted lambs for post mortem examination. C. burnetii infection was confirmed in placental tissue from aborted goats by immunohistochemistry. The first abortions were noticed in week 15 (mid April) 2008. No other farms in the area reported abortions in the weeks prior to the outbreak. At the time of the visit by the Animal Health Service on 15 May (week 20), 40 out of 120 pregnant goats had aborted (33\%), 20 (17\%) had an uncomplicated pregnancy and got healthy offspring, while another 60 animals still had to deliver. No veterinary problems were reported by the other farms. A pet farm in the part of the city where most of the cases resided was visited by the Food and Consumer Product Safety Authority. Three tested goats and one sheep had a weak positive PCR (only one of three Coxiella specific genomic targets positive). However these animals had not been pregnant recently. Hygiene standards at the pet farm were good and none of the employees of the pet farm developed symptoms or had been diagnosed with acute Q-fever. It was concluded that these animals were not the main source of the large cluster of human cases.

\section{Meteorological data}

With an east to north-eastern wind most of the city lies downwind of the dairy goat farm and could potentially have been exposed to contaminated dust particles. During the study period these wind conditions were common in week 16 (14-20 April) with 4 out of 7 days and in week 20 (12-18 May) with 6 out of 7 days (Figure 1).

\section{GIS analysis}

Distance-related attack rates and relative risks for increasingly larger sequential ring buffers from each of

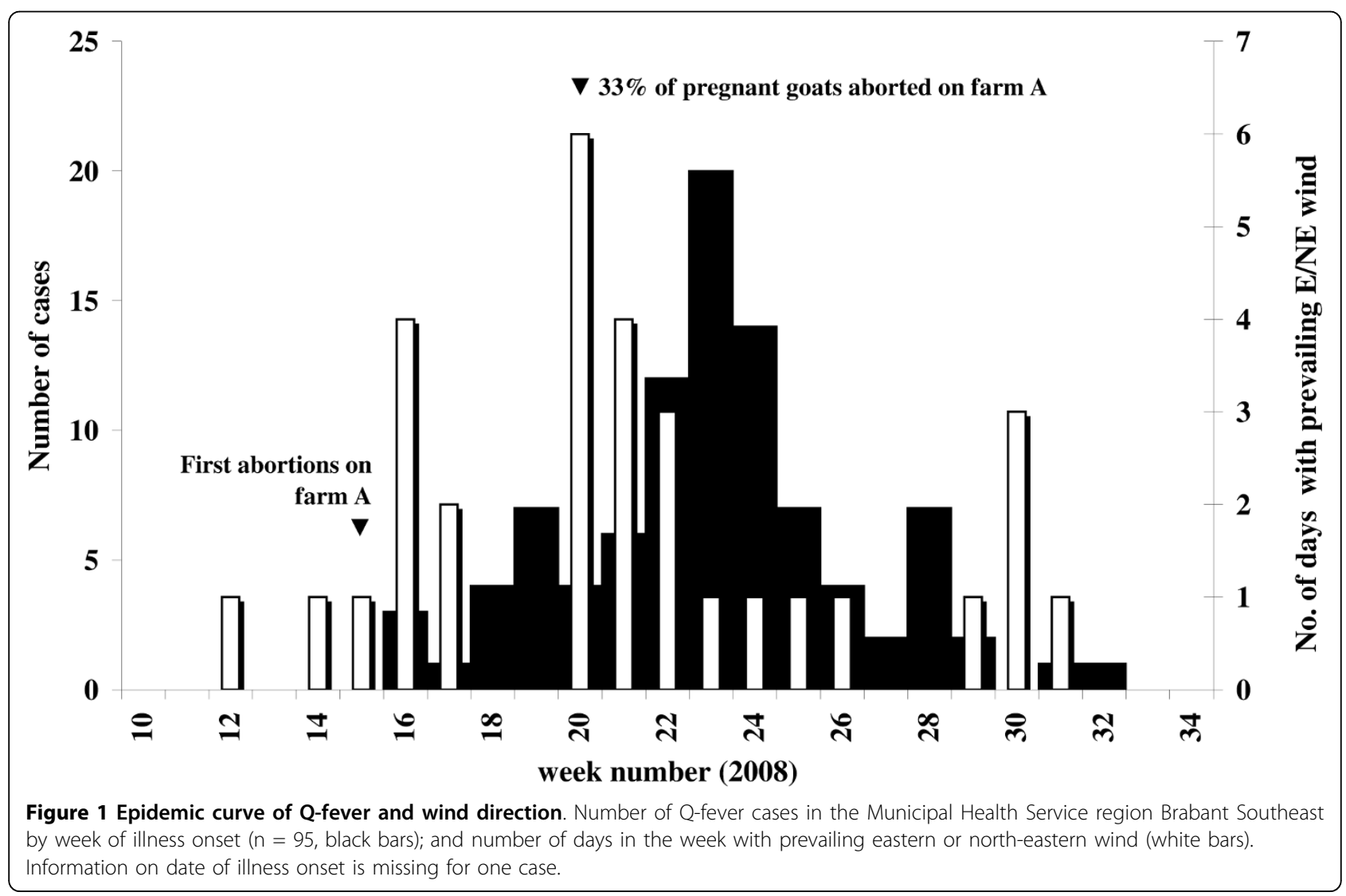




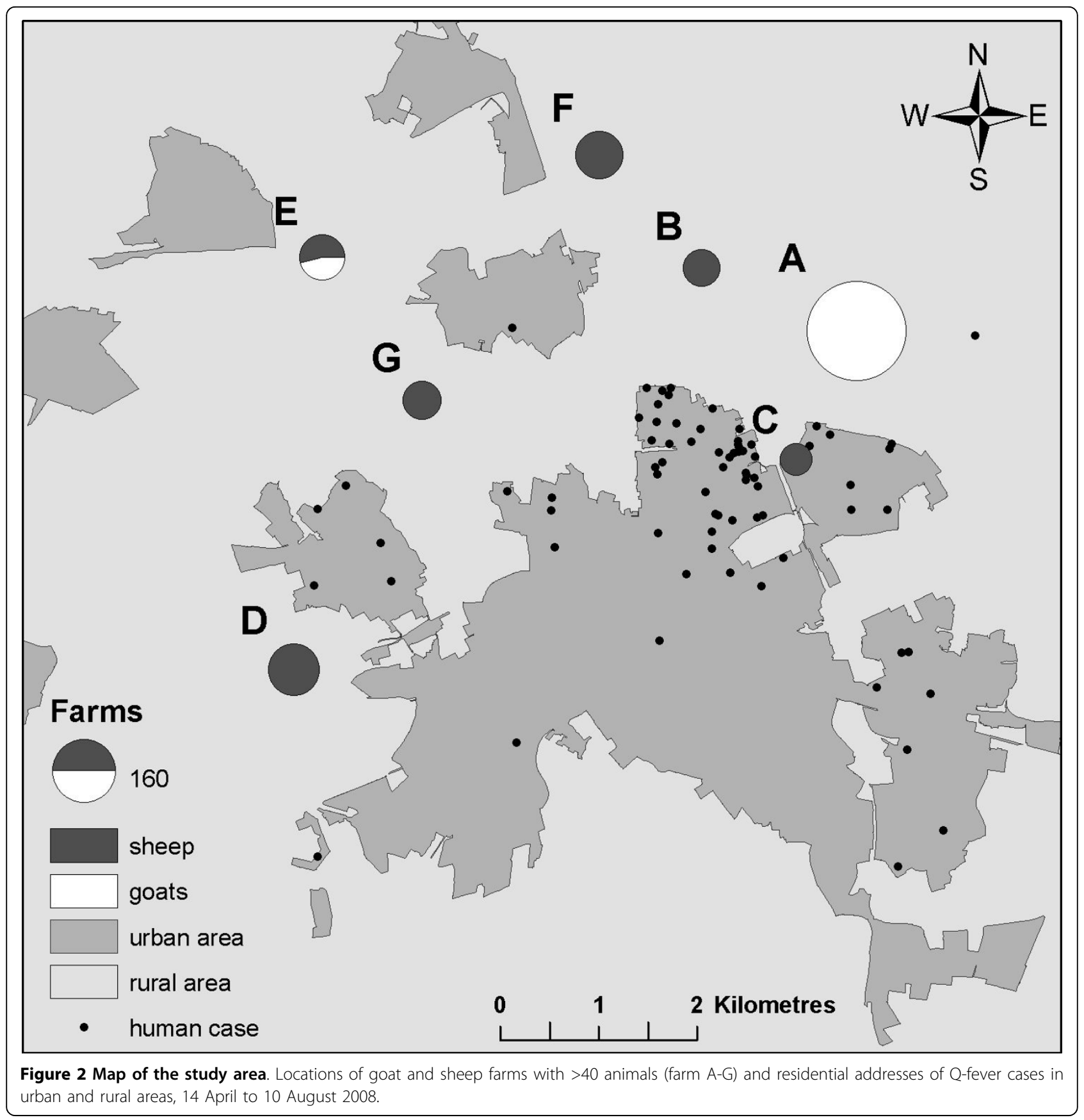

the 7 large farms (A-G) are shown in Table 1. A gradual diminishing relative risk with increasing distance was observed for farm A, the large dairy goat farm with the reported abortion wave, and two small sheep farms $\mathrm{B}$ and $\mathrm{C}$.

Farm A and B both showed a gradual diminishing relative risk with increasing radius, with the exception of the first 1-kilometer zone, possibly due to the small denominator. There were no significant differences in attack rates between farm $\mathrm{A}$ and $\mathrm{B}$. The attack rates within 2 and within 3 kilometres were significantly higher for farm $\mathrm{A}$ than for farm $\mathrm{C}(\mathrm{p}=0.004$ and $\mathrm{p}=$ 0.001 respectively). The highest attack rate for farm $\mathrm{A}$ was for inhabitants residing in the south to south-western direction within 2 kilometres of the farm. Persons living in this zone had a much higher risk for Q-fever than those living more than 5 kilometres away (Relative risk 31.1 [95\% CI 16.4-59.1]). In the 4-5 kilometre zone from farm A, only 1 case was added and the risk of Q-fever for people living in this zone was very low. The 
Table 1 Attack rates of acute Q-fever among residents within circular distance rings around each potential source (A-G) expressed per 100,000 persons over week 16-32 (14 April-10 August 2008), with relative risks (RR) and 95\% confidence intervals $(95 \% \mathrm{Cl})$

\begin{tabular}{|c|c|c|c|c|c|c|c|}
\hline Distance from source & A & B & $C$ & D & $E$ & $\mathbf{F}$ & $\mathbf{G}$ \\
\hline \multicolumn{8}{|l|}{ 0-1 kilometre } \\
\hline Attack rate ${ }^{1}$ & 0 & 0 & 413 & 178 & 0 & 0 & 0 \\
\hline $\mathrm{RR}^{2}(95 \% \mathrm{Cl})$ & 0 & 0 & $53(24-114)$ & $8(1-61)$ & 0 & 0 & 0 \\
\hline Q-fever cases & 0 & 0 & 35 & 1 & 0 & 0 & 0 \\
\hline Population & 92 & 136 & 8482 & 562 & 119 & 281 & 1444 \\
\hline \multicolumn{8}{|l|}{$0-2$ kilometre } \\
\hline Attack rate & 376 & 352 & 203 & 46 & 0 & 13 & 76 \\
\hline $\mathrm{RR}(95 \% \mathrm{Cl})$ & $31(16-59)$ & $25(13-49)$ & $26(12-54)$ & $2(1-5)$ & 0 & $1(0-5)$ & $5(2-13)$ \\
\hline Q-fever cases & 33 & 27 & 59 & 6 & 0 & 1 & 8 \\
\hline Population & 8788 & 7671 & 29,098 & 13,022 & 9852 & 7632 & 10,582 \\
\hline \multicolumn{8}{|l|}{ 0-3 kilometre } \\
\hline Attack rate & 241 & 228 & 137 & 28 & 19 & 69 & 102 \\
\hline RR $(95 \% \mathrm{Cl})$ & $20(11-36)$ & $16(9-30)$ & $18(8-36)$ & $1(1-3)$ & $1(0-2)$ & $4(2-8)$ & $7(4-13)$ \\
\hline Q-fever cases & 59 & 58 & 69 & 9 & 4 & 12 & 28 \\
\hline Population & 24,461 & 25,487 & 50,349 & 32,709 & 20,521 & 17,506 & 27,329 \\
\hline \multicolumn{8}{|l|}{ 0-4 kilometre } \\
\hline Attack rate & 124 & 133 & 101 & 26 & 60 & 147 & 98 \\
\hline $\mathrm{RR}(95 \% \mathrm{Cl})$ & $10(6-19)$ & $10(5-17)$ & $13(6-27)$ & $1(1-2)$ & $2(1-4)$ & $8(5-13)$ & $7(4-12)$ \\
\hline Q-fever cases & 70 & 66 & 71 & 15 & 19 & 49 & 62 \\
\hline Population & 56,585 & 49,570 & 70,151 & 58,002 & 31,425 & 33,270 & 63,547 \\
\hline \multicolumn{8}{|l|}{ 0-5 kilometre } \\
\hline Attack rate & 92 & 88 & 86 & 53 & 94 & 100 & 79 \\
\hline RR $(95 \% \mathrm{Cl})$ & $8(4-14)$ & $6(3-11)$ & $11(5-23)$ & $3(2-4)$ & $4(3-6)$ & $5(3-9)$ & $6(3-10)$ \\
\hline Q-fever cases & 71 & 74 & 78 & 51 & 54 & 71 & 72 \\
\hline Population & 77,558 & 84,374 & 90,779 & 96,402 & 57,329 & 70,680 & 91,123 \\
\hline \multicolumn{8}{|l|}{$>5-10$ kilometre } \\
\hline Attack rate & 12 & 14 & 8 & 21 & 25 & 18 & 14 \\
\hline $\mathrm{RR}$ & 1 & 1 & 1 & 1 & 1 & 1 & 1 \\
\hline Q-fever cases & 13 & 13 & 8 & 39 & 40 & 21 & 17 \\
\hline Population & 107,680 & 93,469 & 102,192 & 185,269 & 162,141 & 113,966 & 121,978 \\
\hline
\end{tabular}

${ }^{1}$ Attack rate: number of Q-fever cases per 100,000 population during the outbreak

${ }^{2} \mathrm{RR}=$ relative risk, attack rate divided by the attack rate of the reference category $5-10 \mathrm{~km}$, with $95 \%$ confidence interval $(\mathrm{Cl})$

other farms D, E, F, G had low attack rates close to the farms compared to farms A-C and they did not show a gradual diminishing relative risk with increasing distance and were considered unlikely infection sources.

\section{Discussion}

Within an on-going large epidemic of Q-fever in a province in the south of the Netherlands we identified a distinct urban cluster of notified Q-fever cases. We combined epidemiological data on notified cases, veterinary and meteorological data in a generic geographic information system to analyse this cluster. The study showed that living within 2 kilometres of a dairy goat farm with abortion problems posed a high risk for Qfever. Furthermore, the time period between the duration of the abortion wave on this dairy goat farm and illness onset in the human cases suggests that airborne transmission of $C$. burnetii from the dairy goat farm could have been the cause of this outbreak. This was further supported by predominant easterly winds on a number of days that could have taken contaminated dust particles to the people living southwest from the farm.

Findings from this urban outbreak are consistent with those from the first community Q-fever outbreak in the Netherlands that occurred in a rural area in 2007. In the 2007 outbreak, airborne transmission from nearby small ruminant farms located to the northeast of the village was suspected as the main route, facilitated by a predominant wind direction from the east during a period of extreme dry weather [1].

There are reports from the international literature that C. burnetii particles can spread from farmland over long 
distances, often facilitated by strong winds towards urban areas [5]. In a community outbreak in the United Kingdom cases were identified up to 18 kilometres from the source [6]. Our results suggest a low risk beyond 5 kilometres from the infection source. The role of wind speed and direction is difficult to analyse when a farm is infectious for a longer period of time.

\section{Source detection}

In the high incidence area in the south of the Netherlands there are thousands of locations with goats and sheep in addition to the many cattle, pig and poultry farms. In this environment the investigation and sampling of all potential animal infection sources to explain human clusters is impossible. Goats, sheep and cattle on many farms throughout the country show serological evidence of previous infection with $C$. burnetii. However, based on the experience since 2007, the prevailing opinion among the human and veterinary public health community is that abortion waves at large dairy goat farms play the predominant role in transmission [4]. Abortion rates of more than $5 \%$ on large dairy goat and sheep farms have to be notified since June 2008. However, abortions in deep litter houses with many hundreds of animals might easily go unnoticed. Attack rate analysis as proposed in the present paper could be an additional tool for source detection.

In order to apply this method real-time, detailed geographic information with residence locations of cases and farm locations, and early notification of human clusters by health professionals are required. The complement fixation test is known to be specific but definitive diagnosis may be delayed because often paired samples are required [7]. Increased awareness among patients and physicians, as well as introduction of rapid laboratory assays, such as PCR, may further reduce the delay between onset of illness and diagnosis and notification [8]. Moreover, in June 2008 Q-fever became a veterinary notifiable disease. This legal framework has facilitated communication between the human and veterinary public health sectors. Clearly, it is essential that the MHS can alert general practitioners in a region where there is a small ruminant farm with clinical Qfever. Veterinary information on Q-fever status of farms in the near proximity, for example clinical Q-fever or positive bulk milk status, could facilitate to pinpoint the most likely sources in an exposed area.

The analysis of the 2008 urban cluster was facilitated by the relatively small number of farms in the area. Even so, the attack rate analysis showed similar results for three farms (A-C) that were located very close to each other. The tool can provide only a rough indication but could facilitate efficient source detection by placing animal locations in classes of 'possible' and 'unlikely'.
This would have to be followed by a veterinary risk assessment and possible sampling at suspected farms. Generating the attack rates requires experience in the use of GIS software. When adequate tools for GIS analyses are available, the workload is limited and the procedure can be automated.

\section{Limitations of the study}

This retrospective cohort study was performed in a new endemic area for Q-fever in the Netherlands where dairy goat farming started recently. Q-fever was laboratory confirmed on the newly established dairy goat farm but no systematic environmental sampling took place in the cluster area. Therefore we cannot exclude that other sources might have been present in the same time period in the region.

Most Q-fever infections remain asymptomatic or give aspecific signs and symptoms. Differences in diagnostic testing and alertness of general practitioners might have led to ascertainment bias. To provide a reliable estimate of the attack rate all patients with symptoms compatible with Q-fever would have to be tested or population surveys would have to be done to include asymptomatic cases.

\section{Research priorities}

Conclusive evidence of a link between certain animals and human Q-fever cases can only be provided by genetic analysis of human, animal and environmental samples. Current typing techniques provide insufficient contrast and methods for culturing and sequencing carry bio safety concerns and are still under development. The presented attack rate analysis assumes homogeneity in the distribution of Coxiella in each concentric ring. In future analyses landscape features should be taken into account to make a more informative analysis possible. This should be combined with information on movement patterns of human cases, recreational activities and other behavioural factors that might change the risk of exposure.

\section{Conclusions}

We found a clear epidemiological link between a cluster of human Q-fever cases and a Q-fever positive dairy goat farm. The present study suggests an effective range of airborne $C$. burnetii spread of $<5$ kilometres. GISbased automated attack rate analysis is a promising tool that could classify animal locations as possible or unlikely infection sources. This methodology should be transformed from a retrospective analysis to a real-time pinpointing tool to guide environmental sampling of potential sources. This requires close collaboration between the human and veterinary public health sectors to ensure timely detection of cases, identification of plausible sources and standardised environmental 
sampling, and application of public health and veterinary preventive measures.

\section{Acknowledgements}

Luppo de Vries and Wouter Boasson at the National Institute for Public Health and the Environment provided technical support for the GIS-based attack rate analysis. We also thank Yvonne van Duynhoven for critically reading the manuscript.

\section{Author details}

${ }^{1}$ Centre for Infectious Disease Control, National Institute for Public Health and the Environment, A van Leeuwenhoeklaan 9, PO Box 1, 3720 BA Bilthoven, the Netherlands. 'Municipal Health Service 'Brabant Zuidoost', Stadhuisplein 2, 5611 EM Eindhoven, the Netherlands. ' Laboratory for Pathology and Medical Microbiology, De Run 6250, 5504 DL Veldhoven, the Netherlands. ${ }^{4}$ Region East, Food and Consumer Product Safety Authority, De Stoven 22, Postbus 202, 7200 AE Zutphen, the Netherlands. ${ }^{5}$ Department of Medical Microbiology and Infection Control, Jeroen Bosch Hospital, Tolbrugstraat 11, PO Box 90153, 5200 ME, 's Hertogenbosch, the Netherlands. ${ }^{6}$ Department of Small Ruminant Health, Animal Health Service, Arnsbergstraat 7, PO Box 9, 7400 AA Deventer, the Netherlands.

\section{Authors' contributions}

$B S, P V$ and $W v d H$ were involved in designing the study and the protocol for data collection. RtS, MW, LZ, AdB and PV provided the human, veterinary and environmental data. BS and TV conducted the data analysis. TV produced the map. BS produced the first draft of the paper, which was revised by WvdH and PS following contributions from RtS, MW, LZ, AdB, TV and PV. All authors read and approved the final manuscript.

\section{Competing interests}

The authors declare that they have no competing interests.

Received: 16 October 2009 Accepted: 16 March 2010

Published: 16 March 2010

\section{References}

1. Karagiannis I, Schimmer B, van Lier A, Timen A, Schneeberger $P$, van Rotterdam B, de Bruin A, Wijkmans C, Rietveld A, van Duynhoven Y: Investigation of a $\mathrm{Q}$ fever outbreak in a rural area of The Netherlands. Epidemiol Infect 2009, 137:1283-1294.

2. Schimmer B, Morroy G, Dijkstra F, Schneeberger PM, Weers-Pothoff G, Timen A, Wijkmans C, Hoek van der W: Large ongoing Q fever outbreak in the south of The Netherlands, 2008. Euro Surveill 2008, 13, pii: 18939.

3. Schimmer B, Dijkstra F, Vellema P, Schneeberger PM, Hackert V, ter Schegget R, Wijkmans C, van Duynhoven Y, Hoek van der W: Sustained intensive transmission of Q fever in the south of the Netherlands, 2009. Euro Surveill 2009, 14, pii: 19210.

4. Brom Van den $\mathrm{R}$, Vellema $\mathrm{P}: \mathrm{Q}$ fever outbreaks in small ruminants and people in the Netherlands. Small Ruminant Res 2009, 86:74-79.

5. Tissot-Dupont H, Amadei MA, Nezri M, Raoult D: Wind in November, Q fever in December. Emerg Infect Dis 2004, 10:1264-1269.

6. Hawker Jl, Ayres JG, Blair I, Evans MR, Smith DL, Smith EG, Burge PS, Carpenter MJ, Caul EO, Coupland B, Desselberger U, Farrell ID, Saunders PJ, Wood MJ: A large outbreak of $Q$ fever in the West Midlands: windborne spread into a metropolitan area? Commun Dis Public Health 1998, 1:180-187.

7. Péter O, Dupuis G, Peacock MG, Burgdorfer W: Comparison of enzymelinked immunosorbent assay and complement fixation and indirect fluorescent-antibody tests for detection of Coxiella burnetii antibody. J Clin Microbiol 1987, 25:1063-1067.

8. Schneeberger PM, Hermans MHA, van Hannen EJ, Schellekens JJA, Leenders ACAP, Wever PC: Real-time PCR on serum samples is indispensable for early diagnosis of acute Q-fever. Clin Vaccine Immunol 2010, 17:286-290.

\section{Pre-publication history}

The pre-publication history for this paper can be accessed here: [http://www.biomedcentral.com/1471-2334/10/69/prepub]

doi:10.1186/1471-2334-10-69

Cite this article as: Schimmer et al:: The use of a geographic

information system to identify a dairy goat farm as the most likely source of an urban Q-fever outbreak. BMC Infectious Diseases 2010 10:69.

\section{Submit your next manuscript to BioMed Central and take full advantage of:}

- Convenient online submission

- Thorough peer review

- No space constraints or color figure charges

- Immediate publication on acceptance

- Inclusion in PubMed, CAS, Scopus and Google Scholar

- Research which is freely available for redistribution

Submit your manuscript at www.biomedcentral.com/submit 